\title{
Rotation of Large Scale Patterns on the Solar Surface as Determined from Filament and Millimeter Data
}

\author{
S. Pohjolainen ${ }^{1}$, B. Vršnak ${ }^{2}$, H. Teräsranta ${ }^{1}$, S. Urpo ${ }^{1}$, \\ R. Brajša ${ }^{2}$, V. Ruždjak², S. Jurač ${ }^{2}, A$. Schroll $^{3}$ \\ ${ }^{1}$ Metsähovi Radio Research Station, 02540 Kylmälä, Finland \\ ${ }^{2}$ Hvar Observatory, 58450 Hvar, Yugoslavia \\ ${ }^{3}$ Sonnenobservatorium Kanzelhöhe, 9520 Sattendorf, Austria
}

\begin{abstract}
The rotation of large scale solar magnetic field patterns was studied using quiescent filaments and low temperature regions observed at $37 \mathrm{GHz}$ as tracers.
\end{abstract}

\section{Introduction}

Solar filaments and low temperature regions (LTR) observed in the millimeter range are located at the inversion lines of the large scale solar magnetic fields pattern (Vršnak et al., 1991). These features can be used as tracers to study the differential rotation on global scale. Filament data were obtained at the Kanzelhöhe Observatory (Austria) and $8 \mathrm{~mm}$ data at the Metsähovi Radio Research Station (Finland). A solar map measured at $37 \mathrm{GHz}$ is presented in Fig. 1.

\section{Differential rotation of filaments from the Kanzelhöhe observations}

The sample of filaments which were used as tracers from the Kanzelhöhe data consisted of 124 polar crown filaments, in order to examine the differential rotation up to a latitude of 80 degrees. The positions of footpoints were measured to trace the rotation. Taking an average footpoint height to be $7000 \mathrm{~km}$ above the photosphere and matching the results with the measurements at low latitudes (Adams and Tang, 1977) we obtained the differential rotation law

$$
\omega=14.45 \pm 0.15-(0.11 \pm 0.9) \sin ^{2} \phi-(3.69 \pm 0.9) \sin ^{4} \phi
$$

which is close to the standard law obtained by other methods. This law includes implicitly the time dependence, since the polar crown shifts towards the pole through the cycle. The differential rotation of filaments is presented in Fig. 2. 


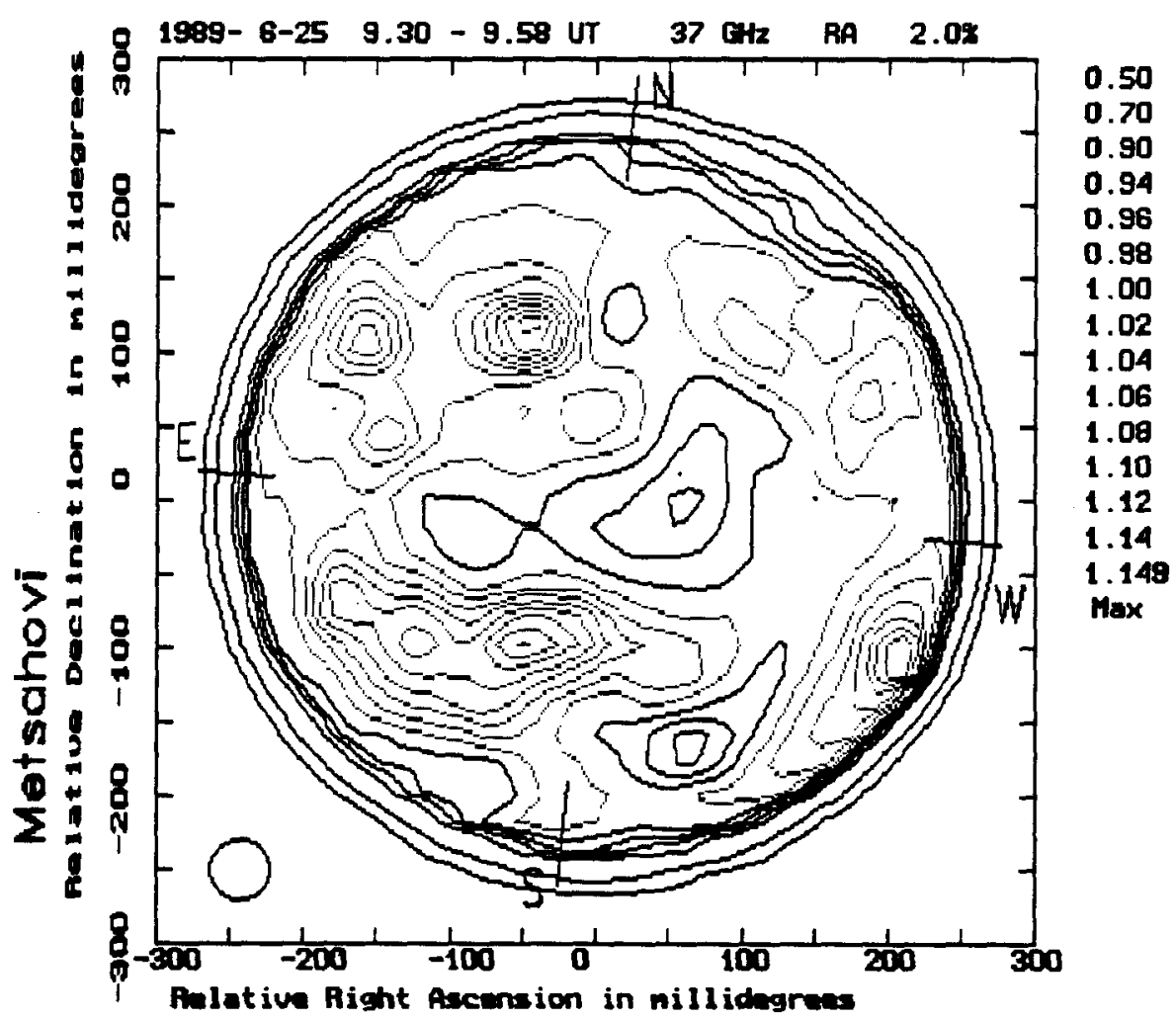

Fig. 1. Solar radio map measured at the Metsähovi Radio Research Station on June 25, 1989 at $37 \mathrm{GHz}(\lambda=8 \mathrm{~mm})$. Black solid lines represent low temperature regions and grey lines active regions, compared to the quiet Sun level (estimation $7800 \mathrm{~K}$ at $8 \mathrm{~mm}$ wavelength).

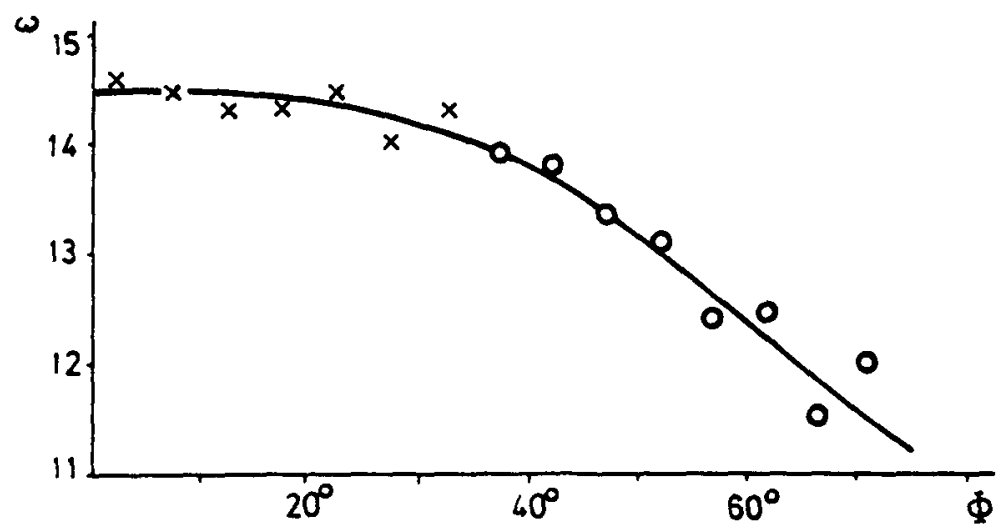

Fig. 2. Sidereal differential rotation $(\omega)$ of filaments (deg/day). Crosses represent the results by Adams and Tang (1977). 


\section{Differential rotation of $8 \mathrm{~mm}$ low temperature regions}

The sample of 214 LTRs covering the years 1979-82 and 1987-89, and latitudes up to 55 degrees, were used as tracers and showed a differential rotation of the form

$$
\omega=14.46 \pm 0.15-(2.91 \pm 0.57) \sin ^{2} \phi .
$$

The rotation curve is presented in Fig. 3a. This is somewhat faster at higher latitudes than in standard laws and implies an effective average height of $15000 \mathrm{~km}$ for the LTRs. However, when we checked the time development of the differential law we found statistically significant variations: During the maximum activity years $1979 / 80$ and 1988/89 the rotation was almost rigid, and the effective height of LTRs would have to be $30000-40000 \mathrm{~km}$ to explain such behaviour in terms of projection effects. In periods 1981/82 and 1987 (close to the minimum) the rotation was following the standard differential rotation law, implying low heights of about $5000 \mathrm{~km}$ for LTRs. The rotation curves for the separate years are shown in Fig. 3b. The polar crown filaments also showed an increase of differential rotation at high latitudes (Brajša et al., 1989), which could be explained by the same kind of time development since polar crown filaments occupy these latitudes at about solar maximum.
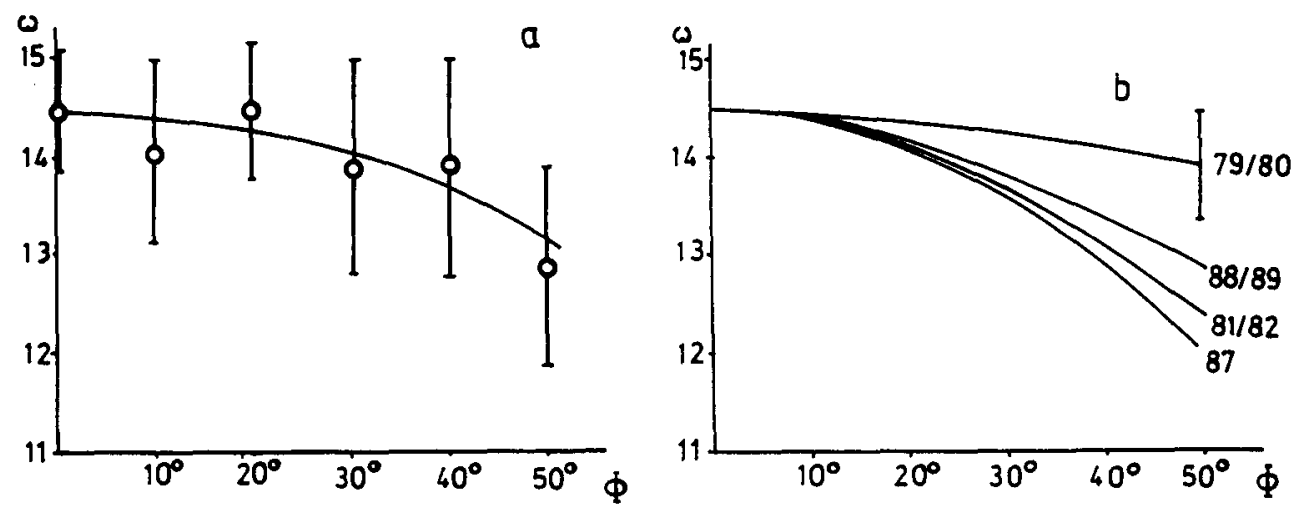

Fig. 3. Sidereal differential rotation $(\omega)$ of LTRs (deg/day). a) The complete sample with error bars representing the standard deviations, b) the time variation with error bar representing the average standard deviation at the latitude of 50 degrees.

\section{References}

Adams, W.M., Tang, F.: 1977, Solar Phys. 55, 499

Brajša, R., Vršnak, B., Ruždjak, V., Schroll, A.: 1989, Hvar Obs. Bull. 13, 449

Vršnak, B., Pohjolainen, S., Teräsranta, H., Urpo, S., Brajša, R., Ruždjak, V., Schroll, A., Jurač, S.: 1991, these Proceedings 